\title{
L'art de faire le vin y su traducción al español: la gestación de un primer vocabulario técnico del vino (1786-1845)
}

\section{$L$ art de faire le vin and its translation into Spanish: the development of the first technical lexicon on wine \\ (1786-1845)}

\author{
MIGUEL IBÁÑEZ RODRÍGUEZ \\ miguel.ibanez@uva.es \\ Universidad de Valladolid
}

Fecha de recepción: 16 de enero de 2017

Fecha de aceptación: 15 de marzo de 2017

Resumen: A partir de 1786 comienzan a llegar a España, en gran medida a través de la traducción, las novedades sobre el Arte de hacer el vino, que habían surgido en Francia y que dan lugar al nacimiento de la enología. Esta disciplina se dota de una terminología de cuya llegada al español y repercusión en las voces tradicionales del vino nos ocupamos en este artículo. Lo hacemos a partir de un corpus de traducciones y textos originales en español comprendidos entre 1786 y 1845, cuyo análisis contrastivo con las fuentes francesas nos permite también estudiar la traducción científico-técnica en esta materia y período.

Palabras clave: Enología, traducción, vocabulario técnico.

Abstract: New knowledge on the art of winemaking arrived in Spain in 1786, mainly through the translation of texts produced in France, cradle of the art that was to give birth to oenology. This paper addresses the arrival of traditional wine words in the Spanish language and the impact thereof. The contrastive analysis of a corpus made up of French texts translated into Spanish and texts originally written in Spanish between 1786 and 1845 allows the study of scientific and technical translation in that field and age.

Keywords: Oenology, translation, technical lexicon. 


\section{INTRODUCCIÓN}

Trataremos de ver en este trabajo en qué medida el Arte de hacer el vino ${ }^{1}$, que había surgido en Francia y que llega a España fundamentalmente a través de la traducción, conlleva la aparición de un vocabulario técnico en el español del vino entre 1786 y 1845. ¿Cómo llegan y cómo se incorporan estos nuevos términos aportados por la nueva disciplina (la enología)? ¿Suponen o no la desaparición del léxico del vino tradicional existente, expresión del saber popular? Nuestra hipótesis de partida es que en el citado período se gesta un primer vocabulario técnico del vino.

Las fechas elegidas vienen determinadas, la de 1786 por ser la del primer texto en el que ya comienzan a llegar a España los nuevos conocimientos enológicos y la segunda, la de 1845, por ser en la que estos ya están plenamente incorporados. Para llevar a cabo nuestra investigación partimos de un corpus de traducciones y originales en español. Al estudiar estos de manera contrastiva en relación a sus fuentes francesas, vamos a contribuir también a un mejor conocimiento de la traducción científicotécnica desde una perspectiva diacrónica. Con sorpresa incluida, al descubrir que uno de los originales seleccionados era en realidad una traducción.

\section{EL CORPUS: L'ART DE FAIRE LE VINY SU TRADUCCIÓN AL ESPAÑOL}

Para elaborar el corpus, nuestras búsquedas se han centrado en los fondos de la biblioteca del Monasterio de Yuso (San Millán de la Cogolla -La Rioja-), del Centro de Documentación de la Cultura del Vino Vivanco (Briones -La Rioja-), de Bodegas El Grifo de Lanzarote y de la Biblioteca Nacional en Madrid.

\subsection{Las fuentes francesas}

El Siglo de las luces también lo es para la vid y el vino (Peynaud, 1996: 135). Son abundantes, en particular en francés, las publicaciones destinadas a mejorar el cultivo de los viñedos y la elaboración del vino, surge el especialista y hay un creciente interés por aprender (Ibáñez, 2015: 277-280). En este contexto aparece en Francia L'Art de faire le vin y parejo a él una nueva ciencia: la enología. En ello fueron figuras determinantes Jean-François Rozier y sobre todo Jean Antoine Chaptal. Los escritos del segundo sobre el vino se incluyen primero en el Cours complet d'Agriculture ou dictionnaire universel del citado Rozier (1800) y después en 1807, bajo el formato de libro, en L'Art de faire le vin, obra cumbre de la materia, que podemos considerar como el primer manual de enología. Se trata del Arte de hacer el vino, la nueva ciencia que "enmienda tan eficazmente a la

\footnotetext{
${ }^{1}$ Conjunto de preceptos y reglas que permiten elaborar el vino con bases científicas.
} 
naturaleza" (Cadet de Vaux, 1803: 52) y cuyos principios van a fijar las reglas de la enología.

El agrónomo y botánico francés Jean-François Rozier, conocido como Abbé Rozier y en España como Abate Rozieres pieza clave en el estudio de la vid y el vino en el siglo XVIII (Ibáñez, 2015: 279). Hemos podido conocer sus obras gracias a Dugour (1800: i-xvi). En 1767 ganó el premio de la Sociedad de Agricultura de Limoges con la que será su primera obra: Mémoire sur la meilleure manière de distiller les vins, et la plus avantageuse relativement à la quantité et à la qualité de l'eau-de-vie. Pocos años después, en $1771^{2}$ fue premiado por la Academia de Marsella por su segunda obra: Mémoire sur la meilleure manière de faire et de gouverner les vins, soit pour l'usage, soit pour leur faire passer les mers que se publicó un año después. En ella J. F. Rozier (1772: 3-4) ya anota que será la química la que puede dar con las claves del vino y su elaboración, la que, en definitiva, podrá establecer el Arte de hacer el vino y su conservación. Aporta una definición del vino, aunque aún seudocientífica (1772: 5): «Le vin tiré du raisin est un composé d'eau, d'air, d'esprit ardent\& de tartre ».

En 1779 decide retirarse a Beziers y consagrar todos sus esfuerzos a los estudios agronómicos y comienza entonces su obra más conocida: « Le cours d'agriculture en forme de dictionnaire » (Dugour, 1800: ix-x). El primer volumen apareció en 1783. La muerte le sorprendió la noche del 28 al 29 de septiembre de 1793 con ocho volúmenes de su obra publicados y dos pendientes cuyos contenidos tenía preparados, pero desgraciadamente se perdieron (Dugour, 1800: xiii-xiv). Es de lamentar la desaparición del artículo dedicado al vino "qu'il avoit entièrement fini, qu'il regardoit comme son meilleur ouvrage... » (Dugour, 1800 : xiv).

Tras su muerte se publican los dos volúmenes restantes. En el último de ellos aparece un extenso artículo dedicado al vino del que se ocupa en 1779 Chaptal, así lo explica años más tarde él mismo en su obra L'Art de faire le vin (Chaptal, 1807: 1). El volumen se publicó al año siguiente y en él ya se sientan las bases de la "théorie de la fermentation" y Chaptal anota como los nuevos conocimientos están dando lugar a una nueva lengua científica y común a todo el sector: "... cette langue scientifique à l'aide de laquelle tous les hommes, tous les pays communiquent entr'eux" (Rozier, 1800: 287).

Más tarde Antoine Alexis Cadet-de-Vaux aprovecha este trabajo para realizar a partir de él una versión de la "doctrina" de Chaptal, con el fin de que llegue a los viticultores. Explica que "le citoyen Chaptal", por entonces

${ }^{2}$ Sin embargo, en la edición que hemos consultado, cuyas referencias bibliográficas aparecen en la relación de obras originales de nuestro corpus, se indica en la portada que el premio lo recibió en 1770. Tanto una fuente como la otra son próximas a los hechos y se contradicen. De todas formas, no es un hecho relevante para nuestro estudio. 
ministro del interior, "a écrit pour les savants et pour les propriétaires, qui doivent à une éducation libérale d'entendre le langage de la science" y que ahora quiere "qu'on écrive pour le vigneron : mission honorable dont ce ministre me charge" (Cadet de Vaux, $1800: 3$ ).

El éxito del artículo vino de Chaptal en la obra de Rozier, en parte debido a la publicación de Cadet de Vaux, llevó al mismo Chaptal (1807: vii) a publicarlo desgajado en formato de libro y así surgió L'Art de faire le vin en 1807, el que se puede calificar como el primer tratado de enológica. Si hacemos un estudio comparativo podemos ver que este tratado tiene básicamente la misma estructura y los mismos capítulos que los publicados en la obra de Rozier en 1800. Ahora bien, cuenta con un mayor desarrollo el capítulo IV dedicado a la fermentación del vino, también incluye un capítulo más, el dedicado a la fabricación del vinagre y el resto tiene mayor extensión, mejor método y claridad, según manifiesta el mismo Chaptal (1807: x) en el "Avertissement" que precede la obra. Y así es efectivamente, cuando en los capítulos repetidos hay actualizaciones estas presentan un mayor rigor científico. En este sentido es ya muy significativo que en los añadidos de la introducción de la obra se mencione por primera vez la nueva ciencia del vino: la enología que, llevada de la mano de la química, va a dotar de nombres técnicos a la vinificación y va a facilitar la comunicación (Chaptal, 1807: 12).

Con esta obra de Chaptal surge la ciencia del vino: la enología. Toda nueva ciencia surge de otra y la enología, hija de la química, no es en ello una excepción. El mismo Chaptal (1807: 10-11) al comienzo de su obra explica que han sido los progresos de la química los que han permitido explicar y fundamentar científicamente los principios que rigen la elaboración del vino: "Or, ces connoissances n'ont été acquises que par les progres de la chimie".

\subsection{Traducciones y originales en español}

¿Cuándo y cómo llega el Arte de hacer el vino a España? Los primeros documentos que hemos encontrado en español datan de 1786 (Ibáñez, 2015: 284-286). Nos referimos en concreto a la Memoria de Francisco Cónsul Jove. Fue presentada por su autor, profesor de física experimental en la Universidad de Santiago de Compostela, el 20 de enero de 1786 en la Sociedad de dicha ciudad. Por azares de la vida una copia manuscrita fue a parar al Archivo del Monasterio de San Millán de la Cogolla (La Rioja) y permaneció silenciada hasta que dio con ella en 2004 el Padre Juan Bautista Olarte, bibliotecario de San Millán. Posteriormente fue publicada por Bodegas David Moreno.

Con ella en la mano nos trasladamos al Centro de Documentación de Vivanco y dimos con la fuente francesa. Si cotejamos esta Memoria con la 
Mémoire de Rozier publicada en París en 1772, podemos ver que esta es la fuente de aquella. ¿Es la Memoria española traducción de la francesa? En rigor no, solo en algunos pasajes lo es. Aunque el texto francés es más extenso que el español, hay coincidencia entre la distribución de los capítulos, así como en sus títulos y en menor medida en los contenidos. De todas formas, Francisco Cónsul se salta la introducción del texto francés y comienza directamente con el primer capítulo. De ella toma la explicación de los distintos tipos de fermentación que va al final de la misma y que introduce el físico español en el capítulo cuarto de su texto. A medida que se avanza en el texto francés, sobre todo a partir del capítulo IV, las correspondencias entre ambos disminuyen. Los capítulos V, VI y VII del original ya no se corresponden con capítulos de la Memoria española, que consta, por otro lado, de seis capítulos o partes. Dado su carácter primerizo, las novedades enológicas no se tratan aquí con la extensión, profundidad y rigor que vemos en otros textos posteriores de nuestro corpus.

En 1803 se publica la traducción de Manuel Pedro Sánchez Salvador y Berrio de L'Art de faire le vin de Cadet-de-Vaux. Su deseo es el de comunicar las nuevas "luces" a todos los cosecheros: "He procurado, que la traducción no desfigure al original, y que su claridad la ponga al alcance de todos los cosecheros" (Cadet de Vaux, 1803: iii-iv).

Juan Álvarez Guerra, por su parte, había iniciado la traducción del Cours complet d'agriculture... ou dictionnaire d'agriculture del Abbé Rozier en 1797 y la concluye en 1803 (Rozier, 1842: 3). Comienza a traducir seis años después de la publicación del primer tomo del original que fue en 1791 y termina tres años después de la edición del último tomo de 1800. En la portada de la versión española se dice que el original ha sido "escrito en francés por una Sociedad de Agrónomos, y ordenado por el Abate Rozier" y que el traductor al castellano es "individuo en la clase de agricultura de la Real Sociedad Económica de Madrid", institución que promovió la traducción a propuesta del mismo Juan Álvarez Guerra. A efectos de nuestro corpus, lo que nos interesa es el último volumen, el XVI, en el que se incluye las entradas de vid/viña (pp. 133-262) y de vino (pp. 292-386).

En 1820 publica Francisco Carbonell y Bravo el Arte de hacer y conservar el vino, que podemos calificar como el primer tratado de enología escrito en español y como tal lo habíamos seleccionado para incorporarlo en nuestro corpus entre las obras originales, pero veremos que no lo es. Señala en la Advertencia del libro (Carbonell y Bravo, 1820: i-ii) que lo contenido en el mismo corresponde a la recopilación de una serie de artículos de la sección de química de la publicación periódica Memorias de agricultura y artes que se venían editando desde 1815. Con el libro dice "pretendo adelantar la industria del suelo español y ser útil a mis conciudadanos" (Carbonell y Bravo, 1820: ii), a sabiendas de la importancia 
que tiene en su "provincia" (Cataluña) y en otras de España la industria del vino.

En ningún momento de su obra señala Carbonell y Bravo que se trata de una traducción (Ibáñez, 2015: 298), sin embargo, nosotros hemos podido comprobar que sí que lo es. Al comienzo de su obra anota únicamente a los autores que le "prestan" los materiales, dando a entender que son sus fuentes y no los textos originales desde los que traduce: Chaptal, François de Neufchateau, Olivier de Serres, Guerin, Chevalier y Remer (Carbonell y Bravo, 1820: 2). Lo que en realidad hace Carbonell y Bravo es traducir descaradamente punto por punto L'Art de faire le vin de Chaptal de 1807. Lo hace desde la página 1 a la 310 , quedando sin traducir dos pasajes: uno de tres páginas (210-213) y otro de siete (pp. 241-218). Carbonell y Bravo no traducen la parte final del libro de J. A. Chaptal, la comprendida entre las páginas 311 y 367 . Teniendo en cuenta esto, tomando como referencia la publicación de L'Art de faire le vin que hemos consultado podemos decir que Carbonell y Bravo traduce el $82 \%$ del libro. Su tratado es más extenso, ya que añade cosas que no están en el original y que, aunque sospechamos que las ha podido traducir de otros textos franceses, no lo podemos afirmar. Carbonell y Bravo añaden la Advertencia (pp. 1-2), el capítulo I (pp. 9-35), el capítulo III (pp. 58-88), el capítulo IV (pp. 110-118), el capítulo V (pp. 147-148), el capítulo VIII (pp. 224-231), el capítulo IX (pp.231-251) y la Noticia acerca de la fabricación del vinagre (pp. 274-289).

Cuando traduce lo hace de manera literal, muy pegado al original y con pequeñas variaciones para acomodar el texto al público español. Por ejemplo, cuando Chaptal se refiere a regiones concretas de Francia, Carbonell las omite sin más: "On a observé, en Champagne, que le raisin cueilli le matin..." (Chaptal, 1807: 103) / "En varias partes se ha observado, que las uvas cogidas por la mañana... (Carbonell y Bravo, 1820: 120). De este modo deslocaliza el texto para que sirva también para el caso español. En alguna ocasión introduce algún ejemplo de su propia región, para buscar cierta adaptación que no llega a conseguirlo ante la gran cantidad de referencias geográficas francesas del original que traslada a su obra: "Así es que las uvas de las orillas del Cher y del Loire en la Turena son muy negras..." (Carbonell y Bravo, 1820: 146).

Años más tarde, en 1842 se volvió a editar la traducción de Juan Álvarez Guerra con el título de Nuevo Diccionario de Agricultura, teóricapráctica y económica y de medicina doméstica y veterinaria. En el prólogo del traductor se justifica la reedición "por haberse hecho sumamente rara" y por "el progreso tan asombroso que han tenido las ciencias exactas" (Rozier, 1842: 3). Juan Álvarez Guerra en esta segunda edición no se incluye en la portada como traductor de la misma; tal vez llevado por la idea de que su trabajo había ido en este caso mucho más allá de la de un simple 
traductor. Tampoco lo niega, pues se desprende de la lectura del prólogo que se trata de una traducción y muestra su respeto al original al tiempo que corrige y añade textos: "aunque corregido y aumentado considerablemente este diccionario, he respetado y conservado el ilustre nombre de su fundador" (Rozier, 1842: 3).

Con relación a esta edición, para el corpus de este trabajo nos hemos ceñido únicamente al artículo consagrado a la vid/viña y al dedicado al vino, ambos aparecen en el volumen XIII y último, publicado en 1845 (Vid/viña pp. 94-164 y vino pp. 182-239). Sus contenidos coinciden en gran parte con los de la primera edición, pero nos ha parecido conveniente incluirlos para verificar la evolución del léxico técnico del vino.

Ahora bien, para poder valorar mejor la llegada de los nuevos tecnicismos hemos querido tener presente un estadio anterior del español del vino. Con ese fin hemos incorporado a nuestro corpus el Libro Segundo, dedicado a la vid y al vino, del tratado Agricultura general de Alonso de Herrera escrito en 1513, el primero en lengua castellana sobre la materia y que fue el tratado de referencia en España.

Para nuestro corpus hemos incluido la edición de 1620, por ser la más antigua conservada, publicada en Madrid por la viuda de Alonso Martín. Según Eloy Terrón esta reimpresión tomó como "base la última edición corregida por el autor, la de 1539" (Alonso de Herrera, 1996: 4). Y también tendremos en cuenta otra edición posterior, la de 1645 publicada en Madrid por Carlos Sánchez.

Igualmente incluimos en nuestro corpus la versión actualizada en 1818 de dicho tratado. Se vuelve a editar en ese año incorporando los nuevos conocimientos agronómicos. En el prólogo Simón de Rojas Clemente habla del éxito de la obra: «lo vertieron a sus idiomas y al latino, y lo transformaron de mil maneras y bajo mil títulos en infinitas ediciones" y según él será después del Quijote, lo obra española más publicada que era consultada en España y en el extranjero a comienzos del siglo XIX, cuando se estaba gestando la ciencia enológica (Alonso de Herrera, 1818-1819: 16). A nosotros nos interesa porque en las adiciones de esta edición actualizada se van a incluir un buen número de tecnicismos ya sancionados por las obras modernas.

Al final del trabajo damos relación detallada de las obras que constituyen nuestro corpus.

\section{HACIA UN ESPAÑOL CIENTÍFICO DEL VINO}

Durante el período estudiado el vocabulario especializado sobre el vino, de carácter tradicional y patrimonial, se va enriqueciendo con el aporte de nuevas voces técnicas, llegadas en buena medida a través de las traducciones del francés. 
En la traducción de Manuel Pedro Sánchez se dice que "solo al sabio pertenece remontar a la causa de estos fenómenos, y solo el hombre que ha tenido una buena educación podrá entender el lenguaje de las ciencias" (Cadet de Vaux, 1803: 109). En las "Observaciones generales" del artículo vino de la traducción de Juan Álvarez Guerra se indica que antes se desconocía "la ciencia", "la teoría de la fermentación" y el "análisis de los vinos" y que a estos conocimientos "debemos los principios invariables que deben guiar al labrador en las operaciones del arte de hacer el vino" y que a dichos conocimientos "debemos esta lengua científica, mediante la cual todos los hombres y todos los países se comunican entre sí" (Rozier, 1803: 296). Estas ideas están en ambos casos en los originales franceses y los traductores lo que hace es trasladarlas al español. De todas formas, al menos el traductor Juan Álvarez Guerra no lo hace de manera pasiva.

La idea de la existencia de una lengua científica con una terminología nueva va ir cuajando en los ilustrados españoles y así lo muestra Juan Álvarez Guerra. En su "Advertencia del traductor" -ahora son sus propias palabras- que precede el primer tomo de su traducción del Cours complet $d^{\prime}$ 'Agriculture... del Abate Rozier señala que cuando no hay "equivalente en castellano" se toma la libertad de "emplear una voz nueva advirtiéndolo allí mismo" (Rozier, 1797: iii). También dice que se inclina por el nombre científico, sin olvidarse de los vulgares (Rozier, 1797: iv). Es una manera de normalizar un vocabulario técnico, que a falta de "obras de agricultura y jardinería" había llevado a que en "cada provincia se haya formado un Diccionario distinto de ella" (Rozier, 1797: iv). Frente a las voces tradicionales con muchas variantes diatópicas van a surgir otras nuevas mejor organizadas, más sistematizadas y que buscarán la precisión y univocidad, aunque no siempre será posible como veremos.

Juan Álvarez Guerra, abogado y político de profesión, además de considerar las nuevas voces con criterio de lexicógrafo, actúa también como traductor profesional sin serlo, pues es consciente de que para traducir el Cours complet d'Agriculture... necesita ayuda del especialista para "corregir y adicionar" y así cuenta con el químico, naturalista, botánico, agricultor, médico, cirujano y veterinario y no duda en añadir algunos artículos de voces desconocidas en la agricultura francesa (Rozier, 1797: ii-iii). En la segunda edición de su traducción se inclina por encabezar sus artículos por la voz más conocida, así lo anota en su prólogo (Rozier, 1842: 3). Otro detalle de su sensibilidad lingüística.

En ocasiones también se evidencia la carencia de terminología. Cosa comprensible para el español de finales del siglo XVIII y comienzos del XIX, que como el resto de lenguas vernáculas, poco a poco va a ir incorporando una terminología especializada de la que hasta la fecha carecía. Carbonell y Bravo (1820: 140) en una nota a pie de página (por lo tanto, no es 
traducción) dice que "empleamos el nombre de alcohol, aunque el principio vinoso de que hablamos parece se diferencia del alcohol" y sin embargo "no tenemos término propio para expresar este principio vinoso".

\subsection{Primeras documentaciones de la voz enología}

Dentro de las nuevas voces está el término enología, que sirve para designar a la nueva ciencia. Los textos que hemos manejado en nuestro corpus contienen las primeras documentaciones en español de esta voz que llega desde el francés y que aparece con distintas grafías incluso dentro de una misma obra.

La primera documentación de este galicismo la hemos encontrado en la traducción de Manuel Pedro Sánchez, en concreto en la carta que precede la obra y que originariamente había escrito en francés Chaptal: "Yo me ocupaba en mi tratado de Enología (o arte de hacer el vino) para nuestros..." (Cadet de Vaux, 1803: viii). A lo largo de toda la traducción aparece en nueve ocasiones más ${ }^{3}$, siempre con mayúscula y con la misma grafía. Es presentada como el "arte de hacer el vino" (p. 44) y como la "nueva doctrina" (p. 76). En la traducción de Rozier (1803: 336) vemos que el traductor la traslada casi tal cual del francés: "La œnologia merece particular atención de parte del labrador, y podemos anunciarle buenos efectos, según la práctica de algunos países...". También aparece en la obra de Carbonell y Bravo (1820: 2), en esta ocasión con acento y con mayúscula: "...la CEnológia, memoria premiada...". Pocas páginas después vuelve a aparecer así escrita œnológia (p. 6 y 8). En esta ocasión en minúscula, cursiva y también con una grafía próxima a la francesa añadiendo un acento. Dos páginas más adelante (p. 10) la volvemos a ver con la misma grafía, pero esta vez sin acento y sin cursiva (œnologia). Resultan sorprendentes estos cambios en tan pocas páginas. Más adelante aún la vemos así escrita onologia (Carbonell y Bravo, 1820: 158).

La vacilación de la grafía es moneda corriente cuando se introduce un extranjerismo. Gómez de Enterría y Gallardo (2010: 68) señalan que cuando una palabra se siente como nueva se suele "conservar con la forma más cercana posible al original". Así lo hace el traductor del Abate Rozier mientras que el de Cadet de Vaux prefiere la adaptación, siendo esta obra cronológicamente anterior.

La enología es presentada como una ciencia, que nace de la química que le "da el nombre que conviene a cada sustancia y a cada operación" propiciando "una comunicación fácil entre todos los agricultores"; lo que hasta ahora no había sido posible pues "cada viñedo tenía su idioma y su método" (Carbonell y Bravo, 1820: 8).

${ }^{3}$ Cadet-de-Vaux, 1803: viii -dos veces-, 44, 71, 72, 76, 80, 82.

Hikma 16 (2017), 9-33 
El DLE explica que enología es de etimología griega, pero por lo explicado aquí llegó al español en 1803 desde el francés a través de la traducción. No aparece en el Diccionario de Autoridades (1726), ni en el Diccionario de Terreros y Pando (1786-1788). En Agricultura general de Alonso de Herrera tampoco está documentada, en ninguna de las ediciones manejadas, ni tan siquiera en la actualización de 1818. Por consiguiente, consideramos que su aparición en la traducción de Manuel Pedro Sánchez de L'Art de faire le vin constituye, sin lugar a dudas, la primera documentación de dicha voz en español.

El primer diccionario que la recoge es el DLE en su edición de 1869 , en él se anota enología, con esta grafía, y se define como "el arte de elaborar los vinos"4. La aparición en un diccionario normativo y general demuestra su aceptación. Así aparece definida hasta la edición de 1925 en la que la voz se explica como "conjunto de conocimientos relativos al vino". En la edición de 1939 sufre una pequeña variación: "Conjunto de conocimientos relativos a la elaboración de los vinos". Y dicha definición ha pervivido hasta la edición actual, la de 2014, que la define exactamente igual.

\subsection{El enologista y el práctico}

Al experto en esta nueva ciencia se le llama enologista: "Cree el C. Perrier, como todo Enologista, que la fabricación de los vinos espumosos..." (Cadet de Vaux, 1803: 81). Se trata de un término también transferido del francés œnologiste, voz formada por derivación mediante el sufijo -iste. Igualmente está documentada, escrito esta vez con minúscula, en la versión actualizada de Alonso de Herrera, (1818: 462, 484, 509). En la primera de estas referencias Chaptal es tildado de "Príncipe de los enologistas". Este contexto, como otros que recogemos más adelante, nos permite matizar que en nuestro corpus el enologista es el experto que escribe sobre la vid y el vino y por lo tanto solo coincide parcialmente con el actual enólogo.

También aparece enologista en la traducción de Rozier (1803: 158) en la entrada vid: "Unos y otros reunidos forman un número que algunos œnologistas modernos no han temido...". Aparece en dicho artículo en tres ocasiones más y siempre con la misma grafía: œnologista ${ }^{5}$ y para referirse también al experto en el viñedo: "Aun no está decidido qual es la estación más favorable para la poda, porque ni los viñeros ni los œnologistas están de acuerdo entre sí sobre este punto" (Rozier, 1803: 220). De igual modo se

${ }^{4}$ La información la tomamos del El Nuevo tesoro lexicográfico de la lengua española (NTLLE), donde están y se pueden consultar las diferentes ediciones del diccionario académico, entre otros. Disponible en http://www.rae.es/recursos/diccionarios/diccionarios-anteriores-17261992/nuevo-tesoro-lexicografico, consultada el 1 marzo de 2016.

${ }^{5}$ Abate Rozier, 1803: 172, 199. 
le aplica al experto en vino, así aparece en la entrada vino: "Los escritos de los œnologistas tienen mil recetas, más o menos buenas, para corregir la acedia de los vinos" (Rozier, 1803: 369). Aquí se corrobora lo que anotábamos más arriba, que el enologista es el teórico del vino y también de la vid.

El enologista no es pues el técnico que elabora los vinos, al que en nuestros textos se le llama práctico: "Finalmente dicen los prácticos, para lograr un buen vino en el trascurso del año, se le sacará..." (Cónsul, 1786: 33). No lo vemos documentado en la traducción de Cadet de Vaux de 1803 y sí en la de Rozier (1803: 332): "Muchos prácticos a quienes les di a probar pensaron lo mismo, y no querían creer que fuese uva verde corregida con azúcar". El DLE lo recoge por primera vez en su edición de 1737 y lo define como "experimentado, versado y diestro en alguna cosa'. En otros casos al técnico se le denomina químico: “.... algunos químicos habían creído que la fermentación espirituosa no podía verificarse..." (Carbonell y Bravo, 1820: 121). En la versión española de Cadet de Vaux (1803: 68) también aparece el químico, como el experto en vinos, "que sabe lo que puede el arte". Igualmente lo vemos documentado en la traducción de Rozier (1803: 324): "Según los experimentos de este mismo químico, parece que el tártaro concurre, lo mismo que el azúcar, a facilitar la formación del alcohol". Lo hemos encontrado en cinco ocasiones más ${ }^{6}$.

En una cita anterior (Rozier, 1803: 220) nos ha aparecido la voz viñero que, a diferencia de enologista, es un neologismo interno formado por derivación de viña, mediante el sufijo -ero. No aparece en Agricultura general de Alonso de Herrera y la primera documentación que hemos encontrado aparece en la traducción de Rozier (1803: 195): "Así que, dexándoles la madera vieja no podemos ser engañados en esto, pues de otra manera sería fácil hacer de un sarmiento largo dos o tres, contra la intención de todo buen viñero". Además de las dos recogidas, aparece en cinco casos más ${ }^{7}$. También aparece en Carbonell y Bravo (1820: 39, 83 y 192). De todas formas, al recogerse en la edición de 1739 del $D L E$ pensamos que es anterior a nuestro corpus. Se define como "el que tiene heredades de viña". Ahora bien, de nuestros textos se desprende que el viñero es también el que trabaja las viñas. En la cita siguiente (Carbonell y Bravo, 1803: 192) se explica que a los viñeros se les da a beber el aguapié, vino de muy poca calidad que solía ofrecerse a los jornaleros: "En varias otras partes toman el orujo sin ventilarle y lo colocan en toneles bien cerrados: echan encima una porción de agua, la cual filtra por entre el orujo, y se carga de un poco de vino que había quedado en este, y sirve de bebida

${ }^{6}$ Abate Rozier, 1803: 267, 276, 320, 325 y 337.

${ }^{7}$ Abate Rozier, 1803: 240, 251, 254, 255 y 265. 
para los viñeros". Los términos práctico, químico, viñero y aguapié son neologismos internos frente a los externos enología y enologista.

\subsection{La voz fermentación aplicada al vino y sus tipos}

La enología surge porque por fin se llega a explicar científicamente el proceso llamado fermentación, por el que el zumo de uva se transforma en vino. La fermentación se explica como algo debido al arte y no a la naturaleza (Carbonell y Bravo, 1820: 98). Es presentada como una nueva teoría: “... mediante la fermentación, cuya teoría y aplicaciones mejor conocidas hoy día que por los antiguos, gracias principalmente a las tareas inmortales de Chaptal..." (Alonso de Herrera, 1818: 462). En torno a ella y sus tipos surge un grupo de interesantes nuevas voces técnicas que vamos a ir desgranando. El término fermentación aplicado al vino encuentra en nuestro corpus las primeras documentaciones. A partir de ahora se diferencia entre la fermentación vinosa (Alonso de Herrera, 1818: 120) y la de otros tipos como, por ejemplo, la fermentación panaria (Alonso de Herrera, 1818: 128). De todas formas, hasta entonces la voz tradicional utilizada había sido cocer, muy frecuente en Alonso de Herrera. Cuando este escribe su Agricultura general en 1513 lo hace en un español que carecía de terminología técnica por lo que tiene que recurrir a voces de la lengua común (cocer) a las que se dota de una nueva acepción especializada. Nos encontramos ante lo que Gutiérrez (2005: 43) Ilama neología de sentido. Veamos algunos ejemplos de cocer. "... y lo echan en sus tinajas a cozer sin casca..." ; "Del cozer ay dos maneras, la una que echan el mosto sin casca a cozer, y desto, se haze el vino claro como agua, y a más dura, y no suele tener tantos vicios"; "Mejor dice el Crecentino que en algunas tierras usan a pisar las uvas y con sus escobajos las echan a cozer en sus tinas..." (Alonso de Herrera, 1620: 40), entre otros ${ }^{8}$.

En la Memoria de Cónsul (1786: 26-27) se encuentran las primeras documentaciones en español de la voz fermentación aplicada al vino. Además, explica los diferentes tipos: "Antes de tratar de las mucosidades de las uvas, explicaremos las especies o grados de fermentación del vino, como son la espirituosa, la ácida y la pútrida". Y se explica cada una de ellas: "El primer grado de fermentación, que es la espirituosa, es la primera alteración sensible que padece el mosto $u$ otro licor que contenga alguna mucosidad dulce, por lo que se convierte en vino". Luego llega la "ácida", calificada como segunda alteración "por la que se mudan los principios y se convierte el vino en vinagre". Y la tercera y última, "la pútrida es la que sigue después de las dos dichas, a la que llamamos corrupción".

\footnotetext{
${ }^{8}$ Más ejemplos en Alonso de Herrera, 1620: 41 y 42.
} 
Más adelante habla de la tumultuosa: "Cuando más está un vino en principiar y completar su fermentación tumultuosa en la cuba tanto más inferior será su calidad y se conservará menos" (Cónsul, 1786: 29). En realidad, es la misma que la espirituosa. También anota otro tipo, la fermentación sensible: "Algunos los llenan solamente tres cuartas partes y los tapan exactamente y luego, por el mes de marzo a agosto, en cuyo tiempo se renueva la fermentación sensible, lo ponen en..." (Cónsul, 1796: 29). En realidad, al hablar de esa renovación de la fermentación sensible, está haciendo referencia a la fermentación que hoy en día se denomina fermentación maloláctica y que en otros textos de nuestro corpus se llama de diferentes maneras: "Hay una fermentación secundaria, que se verifica no en el cubo, sino en las cubas, y es la que perfecciona el vino, llamándose silenciosa, porque es casis insensible" (Cadet-de-Vaux, 1803: 102).

En estas denominaciones hay cierta confusión, comprensible en una Memoria que se hace vagamente eco de la teoría de la fermentación que aún no está plenamente desarrollada. Habla, por ejemplo, de tres tipos de fermentación cuando en realidad es la misma (la fermentación espirituosa, la tumultuosa y la sensible), a la que hoy denominamos fermentación alcohólica o tumultuosa. $\mathrm{Y}$ hace referencia también, sin mencionarla, a la fermentación maloláctica.

En las obras posteriores de nuestro corpus se plantea la fermentación con mayor rigor. En Cadet de Vaux (1803: 10) incluso se define como "el movimiento que se excita en el cubo en que está depositado el fruto, como también en las cubas en que se encierra el licor de las uvas". Está haciendo referencia a los dos tipos de fermentación fundamentales que experimenta el vino: la alcohólica en la vinificación y la maloláctica en la cuba.

En la traducción de Juan Álvarez Guerra se habla de la fermentación insensible: "Recien echado el vino en los toneles se oye un ligero silbido, que proviene del continuo desprendimiento de las burbujitas de gas ácido carbónico que parten de todos los puntos del líquido..." (Rozier, 1803: 357). En realidad, se trata de la continuación de la fermentación alcohólica en fase terminal pues sigue habiendo desprendimiento de gas carbónico.

Observamos falta de precisión y de normalización a la hora de fijar los diferentes tipos de fermentación, lo que suele ser habitual en los inicios de una nueva terminología. Por otro lado, también observamos la pervivencia en los nuevos textos de las formas tradicionales que conviven con las nuevas, como la mencionada cocer. "cocinaría la fermentación ácida con la adición nueva de otro tártaro" (Cónsul, 1786: 34). En la edición actualizada de Alonso de Herrera también hay más ejemplos ${ }^{9}$. Lo vemos también en Carbonell y Bravo, (1820: 124): “... cuando durante el tiempo de cocerse el

${ }^{9}$ Véase Alonso de Herrera, 1818: 462 y 517. 
vino en la tina hay alternativas de frío y de calor..." y también habla de mostro hirviendo (Carbonell y Bravo, 1820: 118). Hervir es también voz tradicional que se usa como sinónimo de cocer. "Este mosto hirviendo imprime el primer grado de calor y añade una porción de mucosidad dulce al mosto..." (Cónsul, 1796: 30). Así pues, las voces tradicionales cocer y hervir no desaparecen por completo y conviven en nuestro corpus con la nueva fermentar.

La fermentación se desencadena por la presencia del fermento o levadura, términos que hay que incluir entre las nuevas voces. Así podemos leer en Alonso de Herrera (1818: 468): “... en que el azúcar del mosto se descomponga y desaparezca por medio del fermento o levadura, convirtiéndose una parte de él en el gas ácido o tufo y la otra en el espíritu del vino...". Observamos en esta cita un fenómeno interesante, el de la rivalidad terminológica, propio de una terminología aún inestable. Por un lado, tenemos "fermento o levadura", dos términos nuevos y por otro "gas ácido o tufo", un término nuevo y otro tradicional, lo que suele ser más habitual en nuestro corpus. Otro ejemplo sería el de "orujo o casca" (Rozier, 1803: 16).

La voz fermentación la encontramos recogida en el Diccionario de Autoridades (1732) definida como "movimiento íntimo de las partículas de cualquier cuerpo, excitado por un fermento extraño...". También está la entrada fermento: "entre los químicos se aplica a cualquier cuerpo activo, que pone en movimiento interior...". El tomo segundo del diccionario de Terreros y Pando (1787) también las incluye. Fermentar la define como "la acción de fermentar" que explica como un término de la física, de la medicina y de la química. De fermento dice este mismo diccionario "término de física, las partes que hacen fermentar, o hinchar y hervir algún cuerpo". En la edición de 1817 del DLE vemos por primera vez la voz fermentar asociada al vino: "moverse o agitarse por sí las partículas de un cuerpo para adquirir nuevas propiedades; como cuando el mosto se hace vino, el vino vinagre, o se pudre algún cuerpo". Sin duda este diccionario se hace eco de lo que ya es moneda corriente en los tratados sobre la vid y el vino. No se recogen, sin embargo, en los diccionarios citados los distintos tipos de fermentación del vino que aquí hemos documentado.

La nueva ciencia explica los elementos que intervienen en la fermentación (mucosidad dulce, principio azucarado, espíritu y alcohol) y el proceso de vinificación al que se asocian voces como descobajar, brisa y flogisto. La óptima madurez de las uvas es imprescindible para que se produzca una buena fermentación y esta se mide por lo que inicialmente se llama la mucosidad dulce "que es la única sustancia fermentable y nutritiva" (Cónsul, 1786: 27). Se está haciendo referencia, sin aún identificarla, al azúcar que es un componente importante de la uva que con la fermentación 
se convierte en alcohol. Esta identificación se produce más adelante cuando ya se habla del principio azucarado en el tratado de Carbonell y Bravo de mayor rigor científico que la Memoria de Cónsul: "Está probado que comparando la naturaleza de todas las sustancias capaces de sufrir la fermentación espirituosa, únicamente las que contienen un principio azucarado son susceptibles de ella;" (Carbonell y Bravo, 1820: 127). Ni mucosidad dulce ni principio azucarado aparecen en el Nuevo Tesoro Lexicográfico de la Lengua Española (NTLLE). No hay ni un solo registro. Esto quiere decir que fueron términos efímeros, reemplazados por la voz azúcar al hacer referencia al mayor o menor grado de dulzor de la uva. De paso azúcar adquiere aquí un uso especializado

\subsection{El espíritu del vino}

En nuestro corpus se habla con frecuencia del espíritu del vino: "... de evitar la pérdida de una gran porción de espíritu de vino, de impedir la..." (Carbonell y Bravo, 1820: 124); también está documentado el adjetivo espirituoso aplicado al vino, del que se dice, por ejemplo, que es un licor espirituoso. ¿A qué se llama espíritu del vino? En algunos pasajes de nuestro corpus parece quedar claro que el espíritu es el alcohol del vino: "...deben producir unos vinos muy espirituosos, supuesto que el azúcar es necesario a la formación del alcohol o espíritu del vino..." (Carbonell y Bravo, 1820: 39). Vemos aquí otro ejemplo de rivalidad terminológica o doblete culto/popular.

Y si vamos a las fuentes francesas a partir de la que se escriben/ traducen los textos de nuestro corpus, aún queda más claro: “... on obtient du vin par la distillation, son esprit ardent ; et cet esprit plus ou moins rectifié par l'application des moyens chimiques, reçoit les noms d'eau-de-vie, d'esprit-de-vin ou alkool" (Chaptal et al. 1801 : v. I p. 2). El vino tenía algo que no se veía, pero se percibía al beberlo, el espíritu, que el químicoenólogo va a identificar con la ayuda del rigor de la ciencia con el alcohol. Para Terreros y Pando (1767), en su tomo segundo, el espíritu del vino es también el alcohol: "Voz de química, y se toma por dos cosas muy distintas, lo primero por un polvo muy fútil, y casi impalpable, y lo segundo por el espíritu de vino tan sutil a fuerza de destilaciones, que no queda parte alguna flemosa". Núñez de Taboada (1825) considera también que el término alcohol pertenece a la química y lo define como: "Licor diáfano e inflamable, que resulta por destilación del vino y de otros licores espirituosos". En Carbonell y Bravo (1820: 84) observamos un uso indistinto de alcohol y de espíritu. Cuando llega el cultismo para sustituir a la voz tradicional la aceptación de aquel suele ser lenta indican Gómez de Enterría y Gallardo (210: 66). 


\subsection{La vinificación}

Mediante la fermentación el mosto se convierte en vino, proceso que pasa a denominarse con el galicismo vinificación: "... puse ese quinto cubo, como habíamos convenido, en las circunstancias menos favorables a la vinificación” (Cadet de Vaux, 1803: 60). En el original se dice: “...je mis cette cinquième cuve, ainsi que nous en étions convenu, dans la circonstance la plus défavorable vinification" (Cadet de Vaux, 1800: 35). No está en Agricultura general de Alonso de Herrera, sí en su actualización de 1818, en una de las adiciones lo podemos leer: ".... para ilustrar más y más el arte sublime de la vinificación, en cuya teoría y práctica somos superiores a los antiguos cuanto es de esperar lo sean a nosotros los enologistas que han de sucedernos" (Alonso de Herrera, 1818: 848) ${ }^{10}$. Este tecnicismo lo vemos recogido por primera vez en Gaspar y Roig (1855) así definido: 'fermentación del mosto de la uva, o transformación del zumo de esta en vino'. El DLE lo incorpora por primera vez en su edición de 1869.

En los textos seleccionados se discute sobre la conveniencia o no de separar el grano del raspón durante la vinificación. Esto genera vocabulario específico que no vemos en los textos de tradición española como el de Alonso de Herrera; nos referimos a desgranar, escobajo o descobajar que si están en los elaborados a partir de fuentes francesas como el de Carbonell y Bravo, (1820: 101) cuando dice "Las uvas blancas no se descobajan" o explica que "las uvas descobajadas daban unos vinos menos espirituosos"11. Sin embargo, no llegan desde el francés. Se forman por derivación de grano y escobajo (estructura del racimo de la que penden las uvas) mediante prefijos y sufijos. Son neologismos internos.

Una vez estrujadas y prensadas las uvas lo que queda es la brisa muy citada en Cadet de Vaux (1803: 62) 12: "... quedando pasmados de ver esto, quando esperaban hallar la brisa enmohecida, y avinagrado el vino". No nos sorprende mucho que no se recoja en la Memoria de Francisco Cónsul, dado su carácter primerizo. Sí que nos parece extraño que no aparezca en las demás obras de nuestro corpus. En Rozier (1803: 275, 316, $317,346,348,356 \ldots)$ se prefiere la forma castiza orujo y en ocasiones casca. Carbonell y Bravo (1820: 77, 78, 103, 104, 106, 108,115, 116...) también prefiere orujo y no usa brisa. Esto nos llevó a pensar que tal vez se trataría de una voz tradicional, lo cual quedó pronto descartado al comprobar que en Agricultura general de Alonso de Herrera no aparece. Se prefiere casca, de la que podemos encontrar muchos ejemplos en la edición de 1620 y en la posterior de 1645: "... quedase la casca para estiércol para

\footnotetext{
${ }^{10}$ Véase también Alonso de Herrera, 1818: 501.

${ }^{11}$ Otro ejemplo en Carbonell y Bravo (1820: 102).

${ }^{12}$ Aparece en diez ocasiones, en las páginas siguientes: $14,46,62,72,73,75,111,117,129$ y

131.
} 
las viñas desque bien podrido..." (Alonso de Herrera, 1620: 40); “... y de las cascas que quedan en las viñas, tienen allí su casa, y en ella bodega pequeña y vasija, y hacen allí aguas ${ }^{13}$ para la gente que labrare la viña" (Alonso de Herrera, 1645: 34). Esto es argumento a favor de que se trata de un tecnicismo que llega con los textos que introducen el nuevo Arte.

Que se trata de un término de nuevo uso se percibe por la necesidad de explicarlo a partir de otro patrimonial como orujo: "... de modo, que el orujo, o brisa caiga, y se hunda...; "... a fermentar con el orujo o brisa de uva negra..." (Cadet-de-Vaux, 1803: 14, 72). En realidad, se trata de un neologismo innecesario, pues la tradición española ya cuenta con la voz casca y también orujo, a pesar de lo cual el traductor de Cadet de Vaux lo usa, alternando ambas, pero no los demás traductores de nuestro corpus. La voz casca es otro ejemplo de voz de la lengua común que adquiere un uso especializado. De igual modo, por ejemplo, que manga como manguera que sirve para sacar el vino de las vasijas: "Se toma una manga o canal de cuero de quatro o seis pies de largo o más, según la magnitud de la vasija, y de dos pulgadas..." (Rozier, 1803: 361). El uso especializado de manga debió producirse a primeros del siglo XIX, pues no lo hemos encontrado documentado con anterioridad.

El DLE recoge brisa por primera vez en su edición de 1726: "El hollejo u orujo que se saca del pie de las uvas después de pisada y exprimida". En el tomo primero de Terreros y Pando (1786) se incluye también y se define así: "Llaman los cosecheros de vino en algunas partes a la casca, que se echa en las cubas, o tinajas para hacer el vino, y que hierva el mosto, y también a la casca en general, que queda pisada ya la uva". La última edición del $D L E$ lo sigue recogiendo con el significado de orujo, aunque no se usa. Explica su etimología a partir del latín hispano brisa y del francés briser. Esto nos lleva a pensar que brisa podría ser también un galicismo, aunque de uso efímero.

Otro ejemplo de tecnicismo registrado en nuestro corpus y que hoy ya no se utiliza es flogisto, incluso de más corto recorrido que brisa; solo lo hemos visto en Cónsul (1786: 32): "Si no han fermentado bastante, su color será oscuro y habrá perdido una parte de aire esencial y de su flogisto...". Aparece definido en Núñez de Taboada (1825) como "elemento hipotético que creía ser el principio de la inflamación de los cuerpos". En el diccionario de Domínguez (1846-1847) se señala que es un término de la química y se define como "Fluido inventado para explicar los fenómenos de la calcinación de los metales, y la combustión de todos los cuerpos". EI DLE, que también lo considera como voz de la química, lo recoge por primera vez en su

${ }^{13} \mathrm{Se}$ usaban las cascas como abono y en otros casos echándoles agua para hacer un vino de mala calidad llamados aguas o también aguapié. 
edición de 1884: "Principio imaginado para explicar la naturaleza del calórico y de la combustión. Todo cuerpo se consideraba compuesto de flogisto y un radical, teoría ya desacreditada". Por los testimonios recogidos parece que tenía poca base científica y eso contribuiría a que dejara de usarse. De todas formas, la actual edición del DLE lo sigue recogiendo y explica que se trata de un helenismo.

\subsection{Voces de la química y de la física}

De antiguo se sabía que durante la fermentación se desprendía un gas, hoy popularmente conocido como tufo, y muchos esfuerzos se hicieron para conservarlo, como el invento de Isabel Gervais ${ }^{14}$, buscando con ello mayor calidad en el vino. Sospechamos que cuando Francisco Cónsul cita en su Memoria el flogisto se refiere al gas que se desprende durante la fermentación del vino y que en la traducción española de Cadet de Vaux se comienza a llamar gas ácido carbónico; en concreto en una nota del traductor Manuel Pedro Sánchez: “... y atrae al instante el gas ácido carbónico, que en su pesadez se eleva poco" (1803: 16). En la traducción de Rozier (1803: 267) se comienza a generalizar ${ }^{15}$ su uso: "... así se observa que en diversas épocas de este movimiento fermentativo hay en él una ligera producción o desprendimiento de gas ácido carbónico" y se le dedica incluso un apartado titulado "Desprendimiento del gas" (pp. 327329). También es frecuente en Carbonell y Bravo (1803: 123): “...el ácido carbónico los arrastra en un estado de verdadera solución". En esta última obra aún perviven denominaciones precientíficas como gas mefítico (Carbonell y Bravo, 1820: 143) y vapor mefítico: “... para estrujar las uvas á fin de poner al vendimiador al abrigo de los vapores mefíticos que se elevan del lagar" (Carbonell y Bravo, 1820: 110). El primer diccionario en recogerlo es el de Núnez de Taboada (1825) y la clasifica dentro de la química y la define así: "formado por la combinación del carbono con el oxígeno". Y a partir de la edición de 1832 se incorporar en el DLE.

Junto al ácido carbónico van surgiendo otros componentes del vino llegados todos ellos desde la química como el ácido tartárico (Cónsul, 1786: 34; Cadet de Vaux, 1803: 102; Rozier, 1803: 269), el ácido málico (Rozier, 1803: 279; Carbonell y Bravo, 1820: 52), ácido hidrosulfúrico (Carbonell y Bravo, 1820: 139, 235) y el ácido acético (Carbonell y Bravo, 1820: 139).

\footnotetext{
${ }^{14}$ Véase la traducción J.C. Choisez, J. C. (1823): Guía indispensable para los cosecheros de vinos, fabricantes de cerveza, de sidra, y para los destiladores de vinos, de granos, de hezes y de todas las materias fermentantes para poder hacer uso con provecho del aparato de la señorita Isabel Gervais. París: Moldes.

${ }^{15}$ Además de este caso podemos encontrar muchos más en las siguientes páginas: 281,320 , 321, 330, 337, 338, 340, 341, 353, 357, 370, 372. 374, 376.
}

Hikma 16 (2017), 9-33 
El doblete del ácido tartárico es alumbre, que aún hoy se conserva en el habla local y que hemos visto en Alonso de Herrera (1620: 43): "Echen un poco de alumbre desecho en el vino, y tomen...". Terreros y Pando también lo recoge (1788) en su tomo tercero: 'es una sal que se eleva en los vinos, y que forma una costra cenicienta que se pega a las paredes de la vasija'.

También hay términos de la física que se incorporan a la enología. Hemos encontrado los siguientes: grado de calor (Rozier, 1803: 269), grado de Reaumur (Rozier, 1803: 271), grado de espirituosidad (Abate Rozier, 1803: 382), fluido gaseoso (Rozier, 1803: 329), peso específico (Rozier, 1803: 134), aire atmosférico (Carbonell y Bravo, 1820: 281), cero en su sentido físico de grado mínimo (Carbonell y Bravo, 1820: 118), cubeta hidropneumática (Carbonell y Bravo, 1820: 122), entre otros. La conocida hoy como cuba hidroneumática es un instrumento de laboratorio que se utiliza para obtener gases por desplazamiento del agua.

Por último, citaremos varios instrumentos de medición como el termómetro de Reaumur (Rozier, 1803:162) ${ }^{16} \mathrm{y}$ el areómetro de Baumé (AbateRozier, 1803: 325), también llamado pesalicor de Beaume (Carbonell y Bravo, 1820: 144). Además de las distintas grafías (Baumé / Beaume) tenemos otro ejemplo de denominaciones diferentes o doblete culto/popular para un mismo instrumento en este caso: areómetro / pesalicor. Hoy en el registro popular se conoce como pesamostos. El DLE recoge areómetro por primera vez en su edición de 1832. Pesalicor no lo vemos en el NTLLE, pero sí en la edición actual del $D L E$.

\section{CONCLUSIONES}

A partir de la primera mitad del siglo XIX, como consecuencia del surgimiento de la enología el ámbito del vino va a disponer de una incipiente lengua técnica común a todo el sector que le va permitir una comunicación fluida entre los distintas regiones y viñedos, salvando así los registros tradicionales que no por ello dejan de existir y convivir con aquella, incluso en la actualidad. Es la química, y en menor medida la física, las que dan origen a la nueva ciencia a la que trasfieren parte de su terminología, en particular la primera. Tránsito que, entre otros motivos, queda testimoniado por el hecho de que a los primeros enólogos se les llame químicos.

Esta lengua técnica común en la que se escriben los primeros tratados sobre la materia surge en francés y desde ella mediante la traducción llega al español confluyendo con la corriente tradicional. Los textos en español, los primeros datan de 1786, en los que se recoge el nuevo Arte de hacer el vino se escriben a partir de fuentes francesas, un buen volumen de ellos son traducciones y otros se actualizan a la luz de los

\footnotetext{
${ }^{16}$ En Cadet de Vaux (1803: 48) aparece con otra grafía: thermómetro.
} 
nuevos conocimientos enológicos surgidos en Francia. También se da el caso de que alguno de ellos presentado como original es en realidad una traducción.

La Memoria de F. Cónsul Jove de 1786 no recoge aún la nueva ciencia, tan solo anota unas primeras pinceladas sobre la misma, procedentes de la fuente francesa a partir de las que está escrita y en parte traducida: la Mémoire de Jean-François Rozier, publicada en 1772 en París. Podemos decir que la fecha clave que marca la llegada de la ciencia enológica a España es 1803, con la traducción de Manuel Pedro Sánchez de L'Art de faire le vin de Cadet de Vaux y la de Juan Álvarez Guerra del artículo vid/viña y sobre todo del dedicado al vino, ambos del tomo XVI del Cours complet d'Agriculture de Jean-François Rozier, en donde se recoge por primera vez en español la teoría de la fermentación. Ahora bien, no es hasta el Arte de hacer y conservar el vino de Carbonell y Bravo de 1820 cuando entra de pleno y con mayor rigor la ciencia enológica en España. Esta obra es un falso original que en realidad es traducción de L'Art de faire le vin de Chaptal publicado en París en 1807. A pesar de que Juan Álvarez Guerra presenta el Nuevo diccionario de agricultura de 1845 como novedoso con relación a la edición de 1803 , no es así si comparamos ambas ediciones, al menos en los artículos vid y vino.

Si comparamos las traducciones con sus originales comprobamos que con frecuencia cuentan con añadidos, supresiones y actualizaciones. Esto nos lleva a concluir que para los traductores lo importante es comunicar los avances de la nueva ciencia, dejando aparcadas otras cuestiones como la fidelidad al texto de partida, no quedando en ocasiones claro lo que se traduce de lo que es escrito original.

El hecho de que el nuevo Artellegue de manera progresiva tiene su repercusión en la terminología, ambigua y vacilante al principio, como ocurre con las denominaciones de los distintos tipos de fermentación en la Memoria de Francisco Cónsul, y mucho más estable y precisa en el Arte de hacer y conservar el vino de Carbonell y Bravo, dado su mayor rigor científico.

El francés, portador del saber enológico, se convierte en lengua dominante con relación a la española, receptora de las nuevas voces técnicas que dan origen a un buen número de galicismos como enología, enologista o vinificación, entre otras. Este mismo fenómeno, que queda patente en nuestro trabajo para el caso del español, es bien seguro que podría evidenciarse para otras lenguas como el italiano o el portugués, por ejemplo.

La llegada de las nuevas técnicas fuerza indirectamente al español del vino a generar nuevas voces propias o neologismos internos. Estos surgen por lo general dotando de usos especializados a palabras comunes 
como manga por manguera o aguas por vino de poca calidad o por derivación como viñero, escobajo o descobajar.

La voz enología, que sirve para denominar a la nueva ciencia, aparece documentada por primera vez en español en 1803, en la traducción de Manuel Pedro Sánchez. En este caso, como en otros muchos, llega transferido desde el francés. La aparición de las nuevas voces no implica la desaparición de las tradicionales con las que van a convivir. Se produce así un curioso fenómeno, el del doblete culto/tradicional como el de alcohollespíritu, gas carbónico/tufo o brisa/orujo.

La nueva corriente del español del vino, expresión del saber científico, que se va generando de manera progresiva se instala con fuerza en el registro escrito y técnico, quedando poco a poco la corriente tradicional, expresión del saber popular, relegada al ámbito oral y local. Aún hoy en día podemos escuchar en ámbitos rurales de tradición vitivinícola voces como cocer y hervir (por fermentar) o cocedero (por bodega). Incluso en el registro culto se mantiene alguna de ellas como bebidas espirituosas.

Con la aparición de este español del vino técnico se producen una serie de fenómenos lingüísticos propios de un vocabulario en gestación. Al doblete se añade también el hecho de que en ocasiones encontramos distintas denominaciones para un mismo concepto, contribuyendo con ello a la llamada inflación terminológica. Los autores/traductores de nuestros textos no siempre son conscientes de la necesaria normalización de la terminología, así mientras Juan Álvarez Guerra en su traducción prefiere desgranar, F. Carbonell y Bravo usa descobajar, para referirse a la operación consistente en separar los granos del raspón del racimo, lo que hoy se conoce como despalillar. Para el gas que se desprende durante la fermentación del vino hemos llegado a recoger cuatro formas distintas, tres populares (flogisto, vapor mefítico y gas mefítico) y una culta (ácido carbónico). Caótica resulta la clasificación de los diferentes tipos de fermentación: espirituosa, ácida, pútrida, tumultuosa, sensible, insensible, etc. En muchos casos se llama de manera diferente a un mismo tipo, así la fermentación que hoy se conoce como alcohólica se denomina indistintamente espirituosa, tumultuosa y sensible.

Cuando se trasfiere el término hemos observado en algunos casos la tendencia a mantener la grafía francesa, así ocurre con œnologista, que correspondería al actual enólogo. Este término experimenta un cambio semántico. En nuestro corpus sirve para referirse tanto al experto que escribe sobre la vid como sobre el vino, aunque no al que cultiva y elabora. Con el paso del tiempo la voz consolidada es enólogo que solo va a hacer referencia al experto y sobre todo al técnico que se ocupa de la elaboración; para el experto en viticultura ha surgido posteriormente otra forma, la de ampelólogo. La densidad semántica de estas primeras voces se va 
descargando en otras a medida que la disciplina madura y se especializa más. Hoy día en portugués sí que se conserva la voz enologista, que convive con enólogo, con el mismo significado. No ocurre lo mismo ni en español ni en italiano.

En algunos de los términos introducidos vemos vacilación ortográfica, lo que suele ser habitual en estos casos. Es un claro síntoma de la novedad del término. El traductor Manuel Pedro Sánchez prefiere Enología (con mayúscula) y enologista y Juan Álvarez Guerra se decanta por no adaptar y escribe CEnológia, œnológia y œnologista. También con el tiempo se han ido fijando las grafías, así vemos cómo el diptongo "ue" de vidueño documentado en nuestro corpus desaparece en la forma actual viduño.

También hemos detectado la rivalidad terminológica que manifiesta el carácter embrionario de esta terminología, así, por ejemplo: "fermento o levadura", En muchos casos lo que se manifiesta es la necesidad de explicar la terminología por su condición precisamente de nueva, así se dice "la acetificación o formación del vinagre" o "gas carbónico o tufo".

Hemos encontrado términos de vida efímera como mucosidad dulce o principio azucarado muy documentados en los primeros textos de nuestro corpus y que luego son progresivamente sustituidos por azúcar sin más. El corvillo utilizado para cortar los racimos en la vendimia está documentado en nuestro corpus. En la actualidad se conserva en el habla de Almendralejo, pero como herramienta de poda y no de vendimia. En La Rioja se prefería corquete y en Castilla y León garillo, ambos son hoy en día arcaísmos.

A la vista de los rasgos lingüísticos observados en los términos estudiados podemos afirmar que nos encontramos ante un español del vino en gestación, que está dando sus primeros pasos. Efectivamente hay una evolución desde los primeros textos de nuestros corpus a los últimos, pero aun así está lejos de ser una terminología consolidada. Eso si estamos ante el punto de partida del español técnico del vino.

Futuras investigaciones nos permitirán recomponer la historia de esta lengua de especialidad y ver hasta cuándo sigue mediatizada por la traducción desde el francés. Sospechamos, habrá que verlo, que hasta tiempos recientes.

\section{CORPuS}

Originales en francés

ROZIER, Jean-François, abbé, Mémoire sur la meilleure manière de faire et de gouverner les vins, soit pour l'usage, soit pour leur faire passer les mers, Lausanne : Chez L. Rosset à Lyon y Chez Le Jay à Paris, 1772. 
-, Cours complet d'Agriculture. Théorique, pratique, économique et de médicine rurale et vétérinaire ou Dictionnnaire Universel d'Agriculture. Paris : Librairie d'Éducation et de Sciences et Arts, 1800. Article Vin (Chaptal) : pp. 284-403.

CADET DE VAUX, Antoine-Alexis, L'Art de faire le vin, d'après la doctrine de Chaptal: instruction destinée aux vignerons. Paris: Bureau de la Décade philosophique, 1800.

CHAPTAL, Jean Antoine, L'Art de faire le vin. Paris: Deterville, 1807.

Traducciones

CADET DE VAUX, Antoine Alexis, Arte de hacer el vino. Traducido por Manuel Pedro Sánchez Salvador y Berrio. Pamplona: Viuda de Longás, 1803.

ROzIER, Abate, Curso completo o diccionario universal de agricultura teórica-práctica, económica y de medicina rural y veterinaria. Traducido al castellano por Juan Álvarez Guerra, individuo de la Sociedad económica de Madrid. Madrid: Imprenta Real, 1803. Tomo XVI; artículo vid/viña (pp. 133-262), artículo vino (pp. 292-386).

CARBonell y BRAvo, Francisco, Arte de hacer y conservar el vino con una noticia acerca la fabricación del vinagre. Barcelona: Antonio Brusi impresor, 1820 [ed. facsímil editada en Barcelona, Dionysos, 1992].

RozIER, Abate, Nuevo diccionario de agricultura, teórica-práctica y económica, de medicina doméstica y veterinaria. Madrid: Boix Editor, 1845. Tomo XIII; artículo vid, viña, pp. 94-164 y vino, pp. 182-239.

Originales en español

CónSUL JOVE, Francisco, Memoria sobre el cultivo de los vinos, sobre los principales progresos de la fermentación vinosa $y$ otros conocimientos y operaciones que dan al vino la mayor perfección y permanencia, 1786.Edición de Juan B. Olarte. Bodegas David Moreno. Badarán -La Rioja-, 2006.

Alonso de HeRRERA Gabriel, Agricultura general. Madrid: Viuda de Alonso Martín, 1620.Libro segundo dedicado a la vid y el vino.

-, Agricultura general que trata de las labranzas del campo, y sus particularidades. Madrid: Carlos Sánchez, 1645. Libro segundo dedicado a la vid y el vino.

—, Agricultura general. Madrid: Imprenta Real,1818. Edición actualizada. Libro segundo dedicado a la vid y el vino.

REFERENCIAS BIBLIOGRÁFICAS: 
Alonso de HerRera, Gabriel, Agricultura general. Edición crítica de Eloy Terrón. Madrid: Servicio de Publicaciones. Ministerio de Agricultura, 1996.

Carbonell y Bravo, Francisco, Arte de hacer y conservar el vino con una noticia acerca la fabricación del vinagre. Barcelona: Antonio Brusi impresor, 1820 [ed. facsímil editada en Barcelona, Dionysos, 1992].

CHAPTAL, Jean Antoine, L'Art de faire le vin. París, Deterville, 1807.

Diccionario de Autoridades. Madrid: Imprenta de Francisco del Hierro, 17261737.

DomíngUEZ, Ramón Joaquín, Diccionario Nacional o Gran Diccionario Clásico de la Lengua Española (1846-47). Madrid-París: Establecimiento de Mellado, 1853.

Dugour, A. J., "Notice sur la vie et les écrits de I'Abbé Rozier". En Abbé Rozier, Cours complet d'Agriculture. T. X. Paris : librairie d'Éducation et de Sciences et Arts ; 1800, pp. I-XVI.

Gaspar Y RoIG, Biblioteca llustrada de Gaspar y Roig. Diccionario enciclopédico de la lengua española, con todas las vozes. Madrid: Imprenta y Librería de Gaspar y Roig, editores, 1855.

GómEZ DE ENTERRÍA, Josefa y GALLARDO, Natividad, "Las versiones de Medicina y Botánica y la nueva terminología científica en el siglo XVIII". En Cuadernos del Instituto Historia de la Lengua, 4, 2010, pp.55-75.

GUTIÉRREZ RODILLA, Bertha, "La creación terminológica: Neología de forma". En El lenguaje de las ciencias, Madrid: Gredos, 2005, pp. 43-55.

IBÁÑEZ RODRíGUEZ, Miguel (2015). "La traducción científico-técnica francésespañol en el ámbito de la enología (1750-1850)". En Çedille, 11, 2015, pp. 273-311.

MuÑoz BELLO, Rosa, "Traducción y enseñanza de la química a finales del siglo XVIII en España". En Brigitte Lépinette y Julia Pinilla Martínez Eds. Reconstruyendo el pasado de la traducción. A propósito de obras francesas especializadas, científicas y técnicas en sus versiones españolas, Granada: Editorial Comares, 2016, pp. 265276.

NúÑEZ DE TABOADA, M., Diccionario de la lengua castellana. 2 vols. París: Seguin, 1825.

El Nuevo Tesoro Lexicográfico de la Lengua Española (NTLLE). Disponible en http://www.rae.es/recursos/diccionarios/diccionarios-anteriores1726-1992/nuevo-tesoro-lexicografico [Fecha de consulta:1 marzo de 2016].

PeYnaud, Émile, Le vin et les jours. Paris: Éditions Payot \& Rivages, 1996.

REAL ACADEMIA ESPAÑola (2014). Diccionario de la lengua española, 23를 ed. Madrid: Espasa. 
ROzIER, Abate, Curso completo o diccionario universal de agricultura teórica-práctica, económica y de medicina rural y veterinaria. Traducido al castellano por Juan Álvarez Guerra, individuo de la Sociedad económica de Madrid. Madrid: Imprenta Real. 16 tomos, 1797-1803.

-, Nuevo diccionario de agricultura, teórica-práctica y económica, de medicina doméstica y veterinaria, del Abate Rozier. Madrid: Boix Editor. 13 tomos, 1842-1845. ${ }^{17}$

Semanario de agricultura y artes, dirigido a los párrocos. Madrid: Imprenta de Villalpando. T. II, 1797.

TERREROS Y PANDO, Esteban, Diccionario castellano con las voces de ciencias y artes. 4 vols. Madrid: Imprenta de la Viuda de Ibarra, $1786-1788 / 1793$

${ }^{17}$ Se trata de una reedición de la obra anterior cuyo traductor es Juan Álvarez Guerra, que en esta ocasión decide no aparecer como tal en la portada, como se explica en el trabajo.

Hikma 16 (2017), 9-33 